\title{
Control of the spontaneous emission from a single quantum dash using a slow-light mode in a two-dimensional photonic crystal on a Bragg reflector
}

\author{
Citation for published version (APA): \\ Chauvin, N. J. G., Nedel, P., Seassal, C., Ben Bakir, B., Letartre, X., Gendry, M., Fiore, A., \& Viktorovitch, P. \\ (2009). Control of the spontaneous emission from a single quantum dash using a slow-light mode in a two- \\ dimensional photonic crystal on a Bragg reflector. Physical Review B, 80(4), 045315-1/5. [045315]. \\ https://doi.org/10.1103/PhysRevB.80.045315
}

DOI:

10.1103/PhysRevB.80.045315

Document status and date:

Published: 01/01/2009

\section{Document Version:}

Publisher's PDF, also known as Version of Record (includes final page, issue and volume numbers)

\section{Please check the document version of this publication:}

- A submitted manuscript is the version of the article upon submission and before peer-review. There can be important differences between the submitted version and the official published version of record. People interested in the research are advised to contact the author for the final version of the publication, or visit the $\mathrm{DOI}$ to the publisher's website.

- The final author version and the galley proof are versions of the publication after peer review.

- The final published version features the final layout of the paper including the volume, issue and page numbers.

Link to publication

\footnotetext{
General rights

- You may freely distribute the URL identifying the publication in the public portal. follow below link for the End User Agreement:

www.tue.nl/taverne

\section{Take down policy}

If you believe that this document breaches copyright please contact us at:

openaccess@tue.nl

providing details and we will investigate your claim.
}

Copyright and moral rights for the publications made accessible in the public portal are retained by the authors and/or other copyright owners and it is a condition of accessing publications that users recognise and abide by the legal requirements associated with these rights.

- Users may download and print one copy of any publication from the public portal for the purpose of private study or research.

- You may not further distribute the material or use it for any profit-making activity or commercial gain

If the publication is distributed under the terms of Article $25 \mathrm{fa}$ of the Dutch Copyright Act, indicated by the "Taverne" license above, please 


\title{
Control of the spontaneous emission from a single quantum dash using a slow-light mode in a two-dimensional photonic crystal on a Bragg reflector
}

\author{
N. Chauvin, ${ }^{1, *}$ P. Nedel, ${ }^{2}$ C. Seassal, ${ }^{2}$ B. Ben Bakir, ${ }^{2}$ X. Letartre, ${ }^{2}$ M. Gendry, ${ }^{2}$ A. Fiore,${ }^{1}$ and P. Viktorovitch ${ }^{2}$ \\ ${ }^{1}$ COBRA Research Institute, Eindhoven University of Technology, P.O. Box 513, NL-5600 MB Eindhoven, The Netherlands \\ ${ }^{2}$ Université de Lyon, Institut des Nanotechnologies de Lyon (INL), UMR CNRS 5270, Ecole Centrale de Lyon, \\ 36 Avenue Guy de Collongue, F-69134 Ecully, France \\ (Received 19 September 2008; revised manuscript received 30 March 2009; published 21 July 2009)
}

\begin{abstract}
We demonstrate the coupling of a single InAs/InP quantum dash, emitting around $1.55 \mu \mathrm{m}$, to a slow-light mode in a two-dimensional photonic crystal on Bragg reflector. These surface addressable 2.5D photonic crystal band-edge modes present the advantages of a vertical emission and the mode area and localization may be controlled, leading to a less critical spatial alignment with the emitter. An increase in the spontaneous emission rate by a factor of 1.5-2 is measured at low temperature and is compared to the Purcell factor predicted by three-dimensional time-domain electromagnetic simulations.
\end{abstract}

DOI: 10.1103/PhysRevB.80.045315

PACS number(s): 78.67.Hc, 42.70.Qs, 12.20.-m

By modifying the local density of optical states (LDOS) around an emitter, its spontaneous emission (SE) rate can be enhanced or suppressed as compared to the vacuum value..$^{1-3}$ This so-called "Purcell effect" can be exploited to funnel most of the spontaneous emission into a single optical mode and is thus crucial for achieving efficient sources of coherent single photons, ${ }^{4,5}$ and more generally in the context of cavity quantum electrodynamics. So far, almost all investigations have focused on modifying the LDOS by fabricating a microcavity around the emitter. Such microcavities have been used to demonstrate the Purcell effect with InAs/GaAs quantum dots (QDs) emitting in the $800-1000 \mathrm{~nm}$ range ${ }^{6-9}$ and more recently around $1300 \mathrm{~nm} .{ }^{10}$ However, modifications of the LDOS can also be obtained in structures supporting propagating modes, such as photonic crystal (PC) wires which have attracted considerable attention recently. ${ }^{11,12}$ Here we report the first demonstration of SE enhancement in a two-dimensional (2D) PC structure on a Bragg reflector (further referred to as a "2.5D PC" structure). ${ }^{13}$ The emitter is represented by a single InAs/InP quantum dash (QDA) emitting around $1550 \mathrm{~nm}$. This structure has two main advantages over standard PC cavities in the context of the targeted quantum photonic devices. First, it radiates around the vertical direction, which is not the case for most of the high- $Q$ factor PC cavities, with the exceptions of the resonators proposed by Kim et al. ${ }^{14}$ and Kang et al. ${ }^{15}$ Second, emitters positioned anywhere in the PC structure can couple to the slow-light mode, which makes it much easier to locate a coupled emitter simply by moving an excitation beam with a diameter of a few micrometers within the structure, whose lateral size is typically of a few tens of micrometers; a similar property is exhibited in the case of PC wire waveguides. ${ }^{12}$ In contrast, in conventional PC cavities only emitters at the center of the cavity can couple to the mode. We note that this paper also represents the first demonstration of Purcell enhancement from a single quantum emitter in the $1550 \mathrm{~nm}$ telecommunication window (in previous investigations on standard PC cavities on InP no Purcell effect was reported $^{16,17}$ ).

The basic photonic design is a high index contrast 2.5D PC resonator, including a graphite 2D PC lattice, drilled in a
$250 \mathrm{~nm}$ InP membrane. The PC structure is designed in such a way as to exploit a low curvature band-edge mode located at the center of the first Brillouin zone, i.e., the $\Gamma$ point. The photonic band structure of such an infinite graphite PC structure was calculated using three-dimensional (3D) plane-wave expansion (PWE) method calculation. The vertical size of the supercell was tuned in order to select the bands that really corresponds to the 2D PC lattice and the result is displayed in Fig. 1. Among the various surface addressable band-edge modes located at the $\Gamma$ point, we exploited the monopolar one (mode M), corresponding to the lower energy flat band. This is a nondegenerated mode where the electromagnetic field is well positioned within the semiconductor, where the QDA are located.

The radiation characteristics of this monopolar mode were

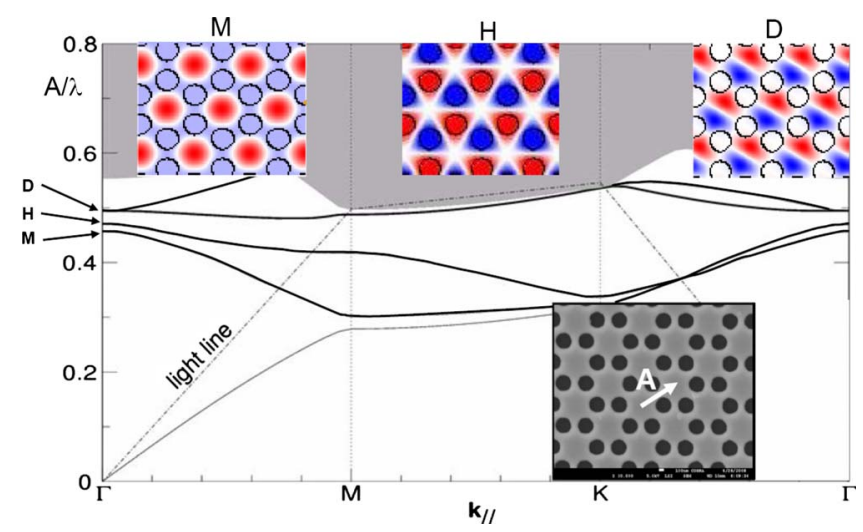

FIG. 1. (Color online) Band diagram of the 2D PC structure. The frequency is expressed in units of $A / \lambda$, for transverse-electric (TE) modes, $A$ being the lattice parameter of the graphite structure. This parameter is related to the nearest holes spacing $a$, corresponding to the lattice parameter of a standard triangular lattice, by the relation $A=\sqrt{3} a$. A scanning electron microscope view of a graphite 2D PC is presented in inset. The three bands indicated in bold correspond to the monopolar $(\mathrm{M})$, hexapolar $(\mathrm{H})$ and dipolar (D) modes of the PC structure. The expected frequencies of these modes are, respectively, $0.45,0.47$, and 0.50 . The corresponding maps of the electromagnetic field ( $\mathrm{Hz}$ component) are indicated in the top insets. 


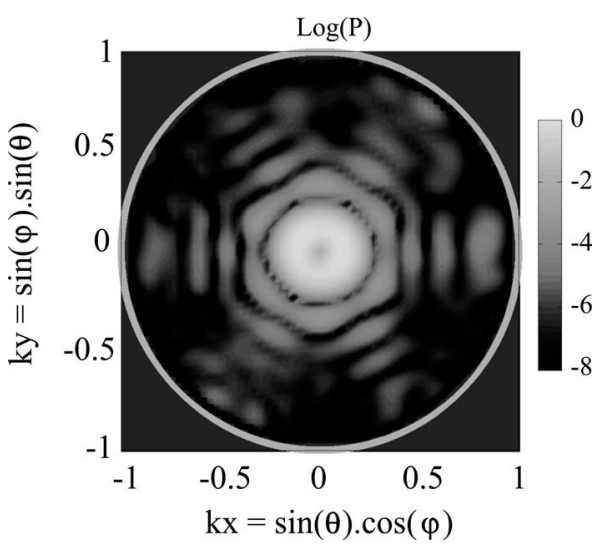

FIG. 2. Radiation diagram (log scale) of the monopolar mode of a graphite 2D PC structure. The circle represents the limit of the light cone.

calculated using the TESSA 3D finite difference in time domain (FDTD) open-source software ${ }^{18}$ for a structure with finite lateral sizes corresponding to a surface of about $76 \mu \mathrm{m}^{2}$. We use a dipole, located into the PC and emitting a temporal pulse, to determine the wavelength of the different resonant modes. Then, using a dipole emitting a cw signal at each resonant wavelength, we identified the different modes (monopolar, hexapolar,...) by their field distribution (the monopolar mode is found at $A / \lambda=0.48)$. Finally, a specific FDTD run is used to calculate the far-field pattern of the monopolar mode. This diagram (in a logarithmic scale in Fig. 2) clearly shows that most of the light in this band-edge mode is radiated vertically. More precisely, $80 \%$ of the light is emitted in an angle of $+/-10^{\circ}$ with regards to the vertical direction. The dip in the vertical direction is a direct evidence of the fact that this wave-guided resonance cannot couple to radiated plane waves precisely at the $\Gamma$ point due to the symmetry of the modes. ${ }^{19}$

The PC slab is positioned on top of a Bragg reflector made with three $\mathrm{Si} / \mathrm{SiO}_{2}$ pairs and which exhibits a reflectance in excess of $98 \%$. We showed elsewhere ${ }^{13}$ that in this configuration, the $Q$ factor of the basic PC resonator may be greatly increased, provided that the PC and the Bragg reflector are separated by a $\mathrm{SiO}_{2}$ layer with an optical thickness $d$ of $3 \lambda / 4$. In this configuration, the directivity of the emission should not be affected; as we showed elsewhere, with $d$ $=3 \lambda / 4 n_{\mathrm{SiO}_{2}}$, the LDOS is higher at $\mathrm{k}_{\|}=0$ (i.e., normal incidence), and therefore, the directivity is even expected to be increased. ${ }^{20}$

In the ideal case of a laterally infinite PC structure, the Purcell factor $F_{p}$ of the optical mode at a given $(\omega, k)$ point of the dispersion characteristics is proportional to the LDOS at $(\omega, k)$. For slow Bloch modes at an extreme of the dispersion characteristics of a 2D PC, we can write $\omega=\omega_{0}+\frac{1}{2} \alpha k^{2}$, $\alpha$ being the photonic band curvature, i.e., its second derivative (in the case of our PC structure, we find, from bandstructure calculation, $\alpha_{\Gamma M} \approx 0.7 \alpha_{\Gamma K}$ in the $k$-space range corresponding to the size of the resonator). In order to give a simple insight into the Purcell effect in such an ideal structure, we will now on consider a 2D isotropic medium, which is close to the real situation at the vicinity of the $\Gamma$ point. The spectral density of states per unit area can be defined by $\frac{d N}{d \omega}=\frac{d N}{d S_{k}} \frac{d S_{k}}{d \omega}$, where $d S_{k}=d k_{x} d k_{y}=2 \pi k d k$ is the unit area in $k$ space. From the dispersion characteristic of the 2D PC, we obtain $\left(\frac{d N}{d \omega}\right)_{\text {membrane }}=\frac{d N}{d S_{k}} \frac{2 \pi}{\alpha}$. Considering the number of optical states in a bidimensional mode, the density of states in $k$-space per unit area is: $\frac{d N}{d S_{k}}=\frac{1}{4 \pi^{2}}$. Thus, the density of states per unit volume is equal to $\left(\frac{d N}{d \omega}\right)_{\text {membrane }}=\frac{1}{2 \pi \alpha h}$, where $h$ is the vertical extension of the mode in the membrane. The Purcell factor is defined by the ratio between the density of states of the membrane and the vacuum density of state and thus a Purcell factor $F_{p} \propto \frac{1}{h \alpha}$ is obtained. The flatter the extreme, the smaller the band curvature and therefore the stronger optical confinement is provided by the $2 \mathrm{D} \mathrm{PC}$, resulting in an increased Purcell factor. Therefore, a key design rule is to select a slow Bloch mode by the $\Gamma$ point that exhibits a curvature as low as possible.

Now, in our situation, the lateral size of the 2D PC structure is finite and the volume of our resonant modes is actually limited by the 2D PC boundaries. A quantitative estimate of $F_{p}$ may be obtained using the classical formula $F_{p}$ $=3 Q \lambda^{3} / 4 \pi^{2} n^{3} V_{m}$. When compared to standard resonant modes with finite group velocity and of similar lifetime, and standing in a cavity of similar size, our resonant modes still exhibit a larger local density of states, this is, due to their envelope function of the electromagnetic field which is not uniform, unlike the case of standard cavity modes with strong lateral confinement. ${ }^{21}$ It can be easily estimated that, for the fundamental laterally confined slow Bloch mode, the Purcell factor can be two to four times larger, if the source is located at the maximum of the electromagnetic field distribution (that is, at any of the antinodes close to the center of the cavity). Another key feature of our resonances is that their emission directivity is maintained, unlike in the case of ordinary cavity modes: this can be easily understood since their radiation pattern is to a large extent determined by their Fourier components in the $k$ space: in the structure presented here, there is one single dominant Fourier component above the light line (albeit a minor one compared to nonradiating components), around the $\Gamma$ point, which essentially contributes to the radiation of the structure in free space.

In order to provide an approximate estimation of the Purcell factor for a structure with a $76 \mu \mathrm{m}^{2}$ surface, and a $19 \mu \mathrm{m}^{3}$ volume, we computed the modal volume of the resonant mode. We found a modal volume $V_{m}=13(\lambda / n)^{3}$. The local pattern of the monopolar mode, its selective location in the semiconductor part of the PC, and the global mode pattern related to the envelope function explain this reduced value of the modal volume. A $Q$ factor of 1700 was also calculated for this resonance. As a result, a Purcell factor $F_{p}=3 Q \lambda^{3} / 4 \pi^{2} n^{3} V_{m} \approx 10$ may be expected for this resonator.

The basic heterostructure has been grown by solid source molecular-beam epitaxy. It includes a single high-density layer of InAs quantum dashes ${ }^{22}$ in the middle plane of the InP membrane. The heterostructure is then transferred onto a Bragg reflector including three pairs of $\mathrm{Si} / \mathrm{SiO}_{2}$ layers, by molecular bonding. The InP subtrate is then chemically removed using a HCl-based etchant. Finally, the PC structure, with a lattice parameter of $775 \mathrm{~nm}$ and a $20 \%$ surface air filling factor, is fabricated by electron-beam lithography and 


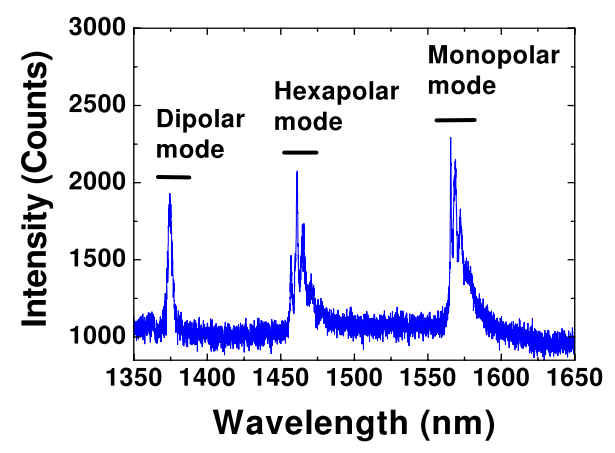

FIG. 3. (Color online) Micro-PL of the 2.5D investigated structure measured at room temperature.

reactive ion etching using a $\mathrm{CH}_{4}: \mathrm{H}_{2}$ mixture. The details on this process can be found elsewhere. ${ }^{23,24}$ The surface of the PC structures is about $200 \mu \mathrm{m}^{2}$, larger than in the simulation, as will be discussed below.

The photoluminescence spectrum of the structure, pumped at $780 \mathrm{~nm}$ and measured at room temperature, is displayed in Fig. 3. The resonant modes could be probed over the whole spectral range between 1350 and $1650 \mathrm{~nm}$. Three groups of sharp peaks clearly appear; each of these corresponds to standing modes formed in the interaction of the Bloch modes in PCs standing around the corresponding wavelength with the edge of the PC area, as described in Ref. 25 . The most intense and sharpest peak of each series corresponds to the highest LDOS of the corresponding Bloch mode, i.e., closest to the $\Gamma$ point. The relative position of the secondary peaks with regards to the main peak is then a clear indication of the band curvature sign of each series. Combining these indications with data from the band structure displayed in Fig. 1, the peaks around $1575 \mathrm{~nm}$ (i.e., $A / \lambda$ $=0.492$ ) are attributed to the monopolar mode, those around $1460 \mathrm{~nm}$ (i.e., $A / \lambda=0.531)$ to the hexapolar mode and finally those around $1375 \mathrm{~nm}$ (i.e., $A / \lambda=0.564$ ) to the dipolar mode. One could note however that, due to an uncertainty in the evaluation of key parameters such as the PC filling factor or the refractive index of the membrane, there is some discrepancy between the wavelength of these peaks and the absolute values of the modes frequencies calculated by PWE (in particular, in the case of the spacing between the $H$ and $D$ modes),

The heterostructure is then studied at liquid-helium temperature. For microphotoluminescence (micro-PL) experiments, the excitation is performed using a continuous-wave diode laser emitting at $860 \mathrm{~nm}$. The excitation was focused on the sample (spot size $4 \mu \mathrm{m}$ diameter) with a microscope objective (numerical aperture 0.5 ). The photoluminescence signal is collected through the same microscope objective and sent to a $1 \mathrm{~m}$ focal length monochromator equipped with a cooled InGaAs photodiode array detector.

The photoluminescence emission of the high-density QDAs at liquid-helium temperature is centered in the 1450$1500 \mathrm{~nm}$ range. To be able to investigate a single QDA, the micro-PL study is performed in the signal tail around 1550 $\mathrm{nm}$ where few QDAs are emitting. Figure 4 shows the micro-PL spectra of a $2.5 \mathrm{D}$ resonant structure for different temperatures, at an incoming excitation power of $500 \mathrm{nW}$.

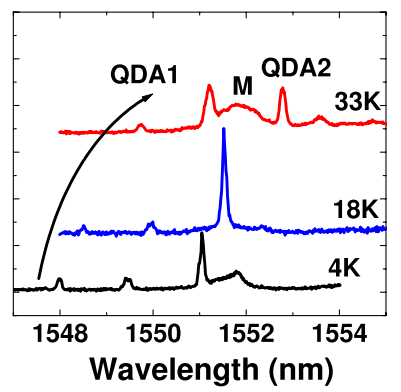

FIG. 4. (Color online) Micro-PL spectra of a $2.5 \mathrm{D}$ photonic crystal structure as a function of the temperature.

The excitation is performed in the vicinity of the center of the PC area. This adjustment of the pumping has been done in order to pump the QD appropriately and optimally.

The three QDA lines are redshifted for increasing temperatures (with respect to $4 \mathrm{~K}$ ) as expected due to the change in the band-gap energy. Three lines are associated to single quantum dash lines due to the fact that a quantum dash exhibits a stronger redshift with temperature than a cavity mode (this latter shifts by $2 \mathrm{~nm}$, slightly less than $0.1 \mathrm{~nm} / \mathrm{K}$ in this range). We focus on lines QDA1 and QDA2 whose measured linewidth is $50 \mu \mathrm{eV}$, a value which is resolution limited. A broad line " $\mathrm{M}$ " is also observed, corresponding to an optical resonance, attributed to the monopolar mode, near the $\Gamma$ point of the PC structure. The observation of the mode despite no coupling with the excitonic lines is attributed to the existence of an unidentified channeling between the QDAs and the off-resonance mode. ${ }^{26}$ At high excitation power, the measured linewidth $(\approx 0.8 \mathrm{~nm})$ corresponds to a quality factor $Q=1800$. One can note that this $Q$ factor is in good agreement with the computed value (1700). For a temperature of $18 \mathrm{~K}$, the spectrum is modified in such a way that the QDA2 line stands at the same wavelength as the slowlight mode M. Our system is then "on resonance," and an enhancement of the QDA intensity by a factor of 2 is observed. The difference of the integrated intensity between off and on resonance has two possible origins: an increase in terms of photon collection efficiency and an increase in the recombination rate due to Purcell effect.

A first experimental evidence of the Purcell effect is obtained by studying the saturation characteristics of the QDA lines on and off resonance. ${ }^{27}$ In order to account for the difference of the integrated intensities due to different extraction efficiencies, the intensity of QDA2 is normalized to have the same intensity as the QDA1 in the linear regime (low excitation intensity). ${ }^{28}$ In Fig. 5(a), when the two QDA are both off resonance (at $T=33 \mathrm{~K}$ ), the same dependence is observed. The off-resonance QDAs have a linear dependence with the excitation power and a saturation is observed for $\mathrm{P}>800 \mathrm{nW}$. This linear dependence confirms the excitonic origin of the QDA lines. At saturation, the two QDA have the same integrated intensity. When the QDA2 is on resonance $(T=18 \mathrm{~K})$, the integrated intensity of the QDA2 at saturation is twice as large as compared the intensity of QDA1 [Fig. 5(b)]. Due to the normalization of the extraction efficiencies, the difference in integrated intensity is due to an enhancement of the spontaneous emission rate of the QDA2. 

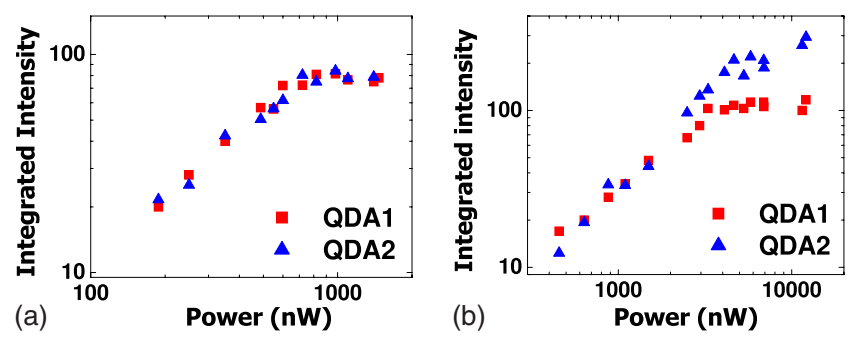

FIG. 5. (Color online) Integrated intensity of the QDA lines: (a) when both lines are off resonance and(b) when QDA1 (2) is off (on) resonance.

Thus a reduction in the lifetime by a factor of 2 can be estimated.

To confirm this result, time-resolved experiments are performed. A $750 \mathrm{~nm}$ pulsed laser is used for the excitation and the photoluminescence signal is coupled into a single mode fiber. Decay times are measured by time-resolved fluorescence spectroscopy using an InGaAs avalanche photodiode ${ }^{29}$ biased above threshold during $100 \mathrm{~ns}$ at a repetition rate of $800 \mathrm{kHz}$, with a dead time of $5 \mu$ s and a quantum efficiency of $10 \%$. The time response of the experimental setup was measured using an InGaAs quantum well emitting at 1300 $\mathrm{nm}$ at room temperature with a lifetime $(50 \mathrm{ps})$ far below the temporal resolution of the detector $(0.6 \mathrm{~ns})$.

The lifetime of the QDAs population located in the bulk (outside the photonic crystal area) is measured by timeresolved photoluminescence using a low incoming excitation power of $15 \mathrm{nW}$ (see Fig. 6). The experimental curve is fitted using the convolution of an exponential decay with the time response of the setup shown in Fig. 6. The lifetime of the QDAs in the bulk is measured using a $1250 \mathrm{~nm}$ high pass filter and an exciton lifetime of $1.78 \pm 0.05 \mathrm{~ns}$ is obtained.

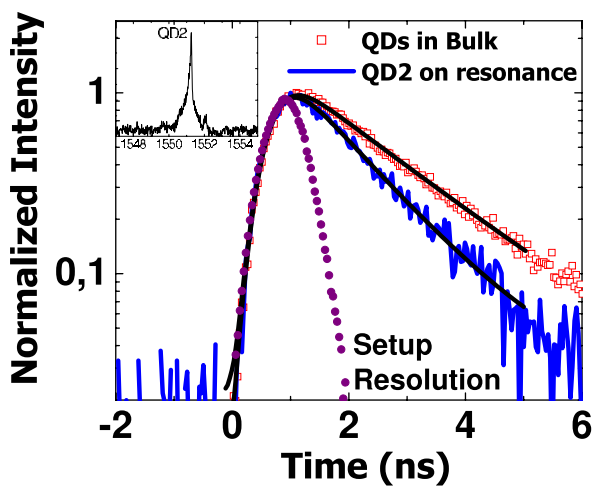

FIG. 6. (Color online) Time-resolved experiments performed at $18 \mathrm{~K}$ on the QDA2 coupled to the PC resonant mode and on a population of QDAs in the bulk. The black curves are the curves fitted using the convolution of a monoexponential decay with the time response.
This value is in the 1.7-2.6 ns range of InAs/InP exciton lifetimes already measured for low excitation power and low temperature. ${ }^{30,31}$ The lifetime of the QDA in resonance with the cavity mode is also measured using a $4 \mathrm{meV}(8 \mathrm{~nm})$ band-pass filter and the same excitation power (at $18 \mathrm{~K}$ ).

The micro-PL spectrum of the QDA at resonance with the mode filtered by the band-pass filter is shown in inset of Fig. 6. A monoexponential decay is observed and a lifetime of $1.20 \pm 0.05 \mathrm{~ns}$ is measured. An increase in the spontaneous emission rate by a factor of $1.5 \pm 0.1$ is observed. As the PL intensity of the on resonance QDA is increased, nonradiative effects can be discarded and the shorter lifetime confirms the Purcell effect. This value is in reasonable agreement with the ratio 2 estimated from the excitation power study. The remaining difference between both values could be explained by a slightly longer lifetime $(\approx 20-30 \%)$ for the offresonance QD as compared with the QDs in the bulk due to the PC band gap.

One could note that the reduction in the QDA lifetime is small compared with the theoretical value of 10 [although significantly higher than the $16 \%$ decrease in the lifetime measured for QDs located inside a PC-based wire and coupled to the zero group-velocity modes (Ref. 12)]. It must be pointed out that the surface of the measured PC structure is 2.5 times larger than the calculated one $\left(200 \mu \mathrm{m}^{2}\right.$ instead of $76 \mu \mathrm{m}^{2}$ ). However, at first order, the Purcell factor does not depend on the surface but only, as explained above, on the curvature of the band. Therefore, the discrepancy can rather be attributed to incomplete spatial and polarization matching of the dash with the $2.5 \mathrm{D}$ resonant mode. Moreover, a fivefold reduction in the Purcell effect between the theoretical calculation and experimental results is in the range of what has already been published on nondeterministic structures. ${ }^{6}$ Much higher values of the Purcell factor are expected if the volume of the band-edge mode is further decreased. A way to achieve this improvement is to control the lateral confinement of light by a local change in the topography ${ }^{32}$ or of the effective index of the PC slab. ${ }^{21}$ This may reduce drastically the modal volume without decreasing the $Q$ factor.

In conclusion, the coupling of a single quantum dash with a slow Bloch mode of a $2.5 \mathrm{D}$ photonic crystal cavity around $1550 \mathrm{~nm}$ is investigated. A slow-light resonance-induced enhancement of the spontaneous emission rate by a factor of $1.5-2$ is observed. This result is promising in view of the realization of higher-efficiency sources of coherent single photons at telecom wavelength.

This work was supported by the European Commission through the IP "QAP" (Contract No. 15848) and the NoE "EPIXNET." J. M. Fedeli and L. Di Cioccio, from CEALETI are acknowledged for Bragg reflector deposition and molecular bonding. Laurent Balet, from COBRA Research Institute, is acknowledged for the realization of the SEM pictures. 
*n.chauvin@tue.nl

${ }^{1}$ E. M. Purcell, Phys. Rev. 69, 681 (1946).

${ }^{2}$ M. Kaniber, A. Kress, A. Laucht, M. Bichler, R. Meyer, M.-C. Amann, and J. J. Finley, Appl. Phys. Lett. 91, 061106 (2007).

${ }^{3}$ B. Julsgaard, J. Johansen, S. Stobbe, T. Stolberg-Rohr, T. Sünner, M. Kamp, A. Forchel, and P. Lodahl, Appl. Phys. Lett. 93, 094102 (2008).

${ }^{4}$ M. Pelton, C. Santori, J. Vuckovic, B. Zhang, G. S. Solomon, J. Plant, and Y. Yamamoto, Phys. Rev. Lett. 89, 233602 (2002).

${ }^{5}$ C. Santori, D. Fattal, J. Vuckovic, G. S. Solomon, and Y. Yamamoto, Nature (London) 419, 594 (2002).

${ }^{6}$ J. M. Gérard, B. Sermage, B. Gayral, B. Legrand, E. Costard, and V. Thierry-Mieg, Phys. Rev. Lett. 81, 1110 (1998).

${ }^{7}$ G. S. Solomon, M. Pelton, and Y. Yamamoto, Phys. Rev. Lett. 86, 3903 (2001).

${ }^{8}$ M. Kaniber, A. Laucht, T. Hurlimann, M. Bichler, R. Meyer, M.-C. Amann, and J. J. Finley, Phys. Rev. B 77, 073312 (2008).

${ }^{9}$ D. Englund, D. Fattal, E. Waks, G. Solomon, B. Zhang, T. Nakaoka, Y. Arakawa, Y. Yamamoto, and J. Vučković, Phys. Rev. Lett. 95, 013904 (2005).

${ }^{10}$ L. Balet, M. Francardi, A. Gerardino, N. Chauvin, B. Alloing, C. Zinoni, C. Monat, L. H. Li, N. Le Thomas, R. Houdré, and A. Fiore, Appl. Phys. Lett. 91, 123115 (2007).

${ }^{11}$ S. Hughes, Opt. Lett. 29, 2659 (2004).

${ }^{12}$ E. Viasnoff-Schwoob, C. Weisbuch, H. Benisty, S. Olivier, S. Varoutsis, I. Robert-Philip, R. Houdré, and C. J. M. Smith, Phys. Rev. Lett. 95, 183901 (2005).

${ }^{13}$ B. Ben Bakir, C. Seassal, X. Letartre, P. Regreny, M. Gendry, P. Viktorovitch, M. Zussy, L. Di Cioccio, and J.-M. Fedeli, Opt. Express 14, 9269 (2006).

${ }^{14}$ S. H. Kim, S. K. Kim, and Y. H. Lee, Phys. Rev. B 73, 235117 (2006).

${ }^{15}$ J. H. Kang, M. K. Seo, S. K. Kim, S. H. Kim, M. K. Kim, H. G. Park, K. S. Kim, and Y. H. Lee, Opt. Express 17, 6074 (2009).

${ }^{16}$ S. Iwamoto, J. Tatebayashi, T. Fukuda, T. Nakaoka, S. Ishida, and Y. Arakawa, Jpn. J. Appl. Phys., Part 1 44, 2579 (2005).

${ }^{17}$ S. Frédérick, D. Dalacu, J. Lapointe, P. J. Poole, G. C. Aers, and
R. L. Williams, Appl. Phys. Lett. 89, 091115 (2006).

${ }^{18} \mathrm{http}$ //alioth.debian.org/projects/tessa/.

${ }^{19}$ K. Sakoda, Optical Properties of Photonic Crystals (SpringerVerlag, Berlin, Heidelberg, 2001).

${ }^{20}$ B. Ben Bakir, Ph.D. thesis, Ecole Centrale de Lyon, 2007.

${ }^{21}$ S. Gardin, F. Bordas, X. Letartre, C. Seassal, A. Rahmani, R. Bozio, and P. Viktorovitch, Opt. Express 16, 6331 (2008).

${ }^{22}$ M. Gendry, C. Monat, J. Brault, P. Regreny, G. Hollinger, B. Salem, G. Guillot, T. Benyattou, C. Bru-Chevallier, G. Bremond, and O. Marty, J. Appl. Phys. 95, 4761 (2004).

${ }^{23}$ C. Seassal, X. Letartre, J. Brault, M. Gendry, P. Pottier, and P. Viktorovitch, J. Appl. Phys. 88, 6170 (2000).

${ }^{24}$ C. Seassal, P. Rojo-Romeo, X. Letartre, P. Viktorovitch, G. Hollinger, E. Jalaguier, S. Pocas, and B. Aspar, Electron. Lett. 37, 222 (2001).

${ }^{25}$ C. Monat, C. Seassal, X. Letartre, P. Regreny, P. Rojo-Romeo, and P. Viktorovitch, M. Le Vassor d'Yerville, D. Cassagne, J. P. Albert, E. Jalaguier, S. Pocas, and B. Aspar, Appl. Phys. Lett. 81, 5102 (2002)

${ }^{26}$ K. Hennessy, A. Badolato, M. Winger, D. Gerace, M. Atature, S. Gulde, S. Falt, E. L. Hu, and A. Imamoglu, Nature (London) 445, 896 (2007).

${ }^{27}$ J. M. Gerard and B. Gayral, J. Lightwave Technol. 17, 2089 (1999).

${ }^{28}$ T. D. Happ, I. I. Tartakovskii, V. D. Kulakovskii, J.-P. Reithmaier, M. Kamp, and A. Forchel, Phys. Rev. B 66, 041303(R) (2002).

${ }^{29}$ C. Zinoni, B. Alloing, C. Monat, V. Zwiller, L. H. Li, A. Fiore, L. Lunghi, A. Gerardino, H. de Riedmatten, H. Zbinden, and N. Gisin, Appl. Phys. Lett. 88, 131102 (2006).

${ }^{30}$ C. Cornet, C. Labbé, H. Folliot, P. Caroff, C. Levallois, O. Dehaese, J. Even, A. Le Corre, and S. Loualiche, Appl. Phys. Lett. 88, 171502 (2006).

${ }^{31}$ E. W. Bogaart, R. Nötzel, Q. Gong, J. E. M. Haverkort, and J. H Wolter, Appl. Phys. Lett. 86, 173109 (2005).

${ }^{32}$ F. Bordas, M. J. Steel, C. Seassal, and A. Rahmani, Opt. Express 15, 10890 (2007). 